\title{
Global analysis of a new nonlinear stochastic differential competition system with impulsive effect
}

\author{
Xuejin LV1 , Lu Wang ${ }^{2}$ and Xinzhu Meng ${ }^{1,3^{*}}$ (D)
}

\author{
"Correspondence: \\ mxz721106@sdust.edu.cn \\ ${ }^{1}$ College of Mathematics and \\ Systems Science, Shandong \\ University of Science and \\ Technology, Qianwangang Road, \\ 279, Qingdao, 266590, P.R. China \\ ${ }^{3}$ State Key Laboratory of Mining \\ Disaster Prevention and Control \\ Co-founded by Shandong Province \\ and the Ministry of Science and \\ Technology, Shandong University of \\ Science and Technology, \\ Qianwangang Road, 279, Qingdao, \\ 266590, P.R. China \\ Full list of author information is \\ available at the end of the article
}

\begin{abstract}
We propose a new stochastic competition chemostat system with saturated growth rate and impulsive toxicant input. The main purpose of this paper is to study the stochastic dynamics of a high-dimensional impulsive stochastic chemostat model and find the threshold between persistence and extinction for the impulsive stochastic chemostat system. First, we investigate the stability of the periodic solution of a deterministic impulsive chemostat model and obtain the threshold between persistence and extinction for the system. Second, by using qualitative analysis method of impulsive stochastic differential equations, we obtain conditions for the extinction and persistence in mean of two microorganisms in the stochastic chemostat model. The results show that a stochastic disturbance or the impulsive effect can cause the microorganisms to go to extinction. Finally, we provide some examples together with numerical simulations to illustrate the analytical results and explain the biological implications.
\end{abstract}

MSC: 60H10;65C30; 91B70

Keywords: stochastic chemostat system; impulsive toxicant input; Itô's formula; persistence in mean; nonlinear growth rate

\section{Introduction}

The chemostat is a kind of experimental device that can be used to cultivate microorganisms and plays an important role in many fields, such as microbiology, ecology, chemical engineering, and so on. Some analysis of a chemostat model and related results can be found in [1-6]. In addition, when microorganism individuals increase greatly, owing to the density-dependent population growth, the effect of saturation growth rate leads to a constant number of microorganism individuals. Comparing with bilinear growth rate, the saturated growth rate may be more suitable for many cases (see, e.g.,[7-9]).

Chemostat models have been applied to open natural environment [1, 2, 4, 7, 10-12]. Environmental pollution by industrial sewage or agricultural pesticides is one of the most serious social and ecological problems. The toxicant in the environment is a threat to the survival of the exposed microorganisms. Therefore, it is of great importance to investigate the effects of toxicant and obtain a theoretical threshold between the extinction and persistence of the microorganisms in a polluted environment [13-15]. In recent years, many

(c) The Author(s) 2017. This article is distributed under the terms of the Creative Commons Attribution 4.0 International License (http://creativecommons.org/licenses/by/4.0/), which permits unrestricted use, distribution, and reproduction in any medium, provided you give appropriate credit to the original author(s) and the source, provide a link to the Creative Commons license, and indicate if changes were made. 
works have been carried out to study the effects of toxicant on biological populations [1618]. In the 1980s, some deterministic toxicant-population models were initially proposed by Hallam $[16,17]$. From then on, many important and valuable deterministic models with toxicant effect were investigated by some scholars [14-17]. However, in the real world, a waste water with toxicant is always input impulsively, and the population system is inevitably affected by an impulsive toxicant input. Some authors have studied the effects of impulsive toxicant input on the persistence and extinction of microorganisms in a polluted environment [18-23].

Chemostat models are inevitably affected by the white noise stochastic disturbance; therefore the dynamics of a stochastic chemostat model may be different from that of a deterministic model. Some scholars have studied the dynamics behaviors of various kinds of stochastic systems and obtained many good results [24-42]. Recently, taking both impulsive toxicant input and white noises into account, persistence and extinction of a singlespecies population system in a polluted environment with random perturbations and impulsive toxicant input were explored [42, 43].

Recently, many scholars focus on the research of impulsive stochastic differential systems. Hence, the asymptotic stability of some impulsive stochastic differential systems were investigated, and many good results were obtained [44-49]. To capture essential features of the processes, the following several aspects should be considered in the formulation of chemostat models: (a) two-species competition for a limiting nutrient supplied at a constant rate; (b) impulsive toxicant input; (c) white noise stochastic disturbance; and (d) saturated growth rate. To our knowledge, there are only a few works that consider the qualitative analysis of high-dimensional impulsive stochastic chemostat competition models with saturated growth rate. Therefore, based on the four aspects, we propose a new competition model with white noise disturbance and impulsive toxicant input. For this new system, we explore the threshold between the extinction and persistence of two microorganisms and study the influences of impulsive toxicant input and stochastic disturbance on system dynamics. A deterministic chemostat competition model with saturated growth rate and pulsed toxicant input can be described by the following impulsive differential equation:

$$
\left\{\begin{array}{l}
\dot{S}(t)=Q\left(S_{0}-S(t)\right)-\frac{\mu_{1} S(t) x_{1}(t)}{\delta_{1}\left(a_{1}+x_{1}(t)\right)}-\frac{\mu_{2} S(t) x_{2}(t)}{\delta_{2}\left(a_{2}+x_{2}(t)\right)}, \\
\dot{x}_{1}(t)=\frac{\mu_{1} S(t) x_{1}(t)}{a_{1}+x_{1}(t)}-Q x_{1}(t)-r_{1} c(t) x_{1}(t), \\
\dot{x}_{2}(t)=\frac{\mu_{2} S(t) x_{2}(t)}{a_{2}+x_{2}(t)}-Q x_{2}(t)-r_{2} c(t) x_{2}(t), \\
\dot{c}(t)=-h c(t), \\
S\left(n \tau^{+}\right)=S(n \tau), \quad x_{i}\left(n \tau^{+}\right)=x_{i}(n \tau) \quad(i=1,2), \\
c\left(n \tau^{+}\right)=c(n \tau)+u, \quad n \in Z^{+},
\end{array}\right\} t \neq n \tau, n \in Z^{+},
$$

where $S(t)$ denotes the concentration of the unconsumed nutrient at time $t, x_{i}(t)$ represents the concentration of the microorganism at time $t(i=1,2), c(t)$ is the concentration of the toxicant in the chemostat at time $t, S_{0}$ and $Q$ are the input concentration of the nutrient and the flow rate of the chemostat, respectively, $\mu_{i}$ is the maximal growth rate (or predation rate), $a_{i}$ is the half-saturation constant $(i=1,2), r_{i}$ is the depletion rate coefficient of the microorganism population due to organismal pollutant concentration, $\delta_{i}$ is the yield of the microorganism $x_{i}(t)$ per unit mass of substrate $(i=1,2), h$ denotes the loss 
rate of toxicant in culture medium of the chemostat, $u$ is the amount of toxicant pulsed each $\tau$, where $\tau$ is the period of pulsing, and all the coefficients are positive constants. The function $\frac{\mu_{i} S(t) x_{i}(t)}{a_{i}+x_{i}(t)}$ represents saturated growth rate showing density effect of the microorganism population, which is different from $\frac{\mu_{i} S(t) x_{i}(t)}{a_{i}+S(t)}$ (see $\left.[6,7,9]\right)$.

Note that all parameters in system (1) can be affected by environmental noise, which always fluctuates around some average values. However, in this paper, we only consider the case that there is randomness involved in the maximal growth rate (or predation rate) $\mu_{i}$, which is one of the crucial parameters, to the culture of microorganism. In this case, $\mu_{i}$ changes to a random variable $\mu_{i}+\sigma_{i} \dot{B}_{i}$, so that $\frac{\mu_{i} S(t) x_{i}(t)}{a_{i}+x_{i}(t)} \rightarrow \frac{\mu_{i} S(t) x_{i}(t)}{a_{i}+x_{i}(t)}+\frac{\sigma_{i} S(t) x_{i}(t)}{a_{i}+x_{i}(t)} \dot{B}_{i}(t)$, where $B_{i}(t)$ is a standard Brownian motion with intensity $\sigma_{i}^{2}>0(i=1,2)$. Then a stochastic version can take the following form:

$$
\left\{\begin{aligned}
\mathrm{d} S(t)= & \left(Q\left(S_{0}-S(t)\right)-\frac{\mu_{1} S(t) x_{1}(t)}{\delta_{1}\left(a_{1}+x_{1}(t)\right)}-\frac{\mu_{2} S(t) x_{2}(t)}{\delta_{2}\left(a_{2}+x_{2}(t)\right)}\right) \mathrm{d} t \\
& -\frac{\left.\sigma_{1} S(t) x_{1}(t)\right)}{\delta_{1}\left(a_{1}+x_{1}(t)\right)} \mathrm{d} B_{1}(t)-\frac{\sigma_{2} S(t) x_{2}(t)}{\delta_{2}\left(a_{2}+x_{2}(t)\right)} \mathrm{d} B_{2}(t), \\
\mathrm{d} x_{1}(t)= & \left(\frac{\mu_{1} S(t) x_{1}(t)}{a_{1}+x_{1}(t)}-Q x_{1}(t)-r_{1} c(t) x_{1}(t)\right) \mathrm{d} t \\
& +\frac{\sigma_{1} S(t) x_{1}(t)}{a_{1}+x_{1}(t)} \mathrm{d} B_{1}(t), \\
\mathrm{d} x_{2}(t)= & \left(\frac{\mu_{2} S(t) x_{2}(t)}{a_{2}+x_{2}(t)}-Q x_{2}(t)-r_{2} c(t) x_{2}(t)\right) \mathrm{d} t \\
& +\frac{\sigma_{2} S(t) x_{2}(t)}{a_{2}+x_{2}(t)} \mathrm{d} B_{2}(t), \\
\mathrm{d} c(t)= & -h c(t) \mathrm{d} t, \\
S\left(n \tau^{+}\right)= & S(n \tau), \quad x_{i}\left(n \tau^{+}\right)=x_{i}(n \tau) \quad(i=1,2), \\
c\left(n \tau^{+}\right)= & c(n \tau)+u, \quad n \in Z^{+},
\end{aligned}\right\} \quad t \neq n \tau, n \in Z^{+}
$$

where $\sigma_{i}$ is the environmental white noise disturbance coefficient $(i=1,2)$.

For convenience of description, we introduce the following definitions: $\left(\Omega, \mathcal{F},\{\mathcal{F}\}_{t \geq 0}, \mathcal{P}\right)$ is a complete probability space with a filtration $\left\{\mathcal{F}_{t}\right\}_{t \geq 0}$ satisfying the usual conditions (i.e. it is increasing and right continuous, whereas $\mathcal{F}_{0}$ contains all $\mathcal{P}$-null sets); $B(t)$ is a scalar Brownian motion defined on this probability space; $S(t)$ and $x_{i}(t)$ are continuous at $t=n \tau$, $c(t)$ is left continuous at $t=n \tau$ and $c\left(n \tau^{+}\right)=\lim _{t \rightarrow n \tau^{+}} c(t)$; and $\langle f(t)\rangle=\frac{1}{t} \int_{0}^{t} f(\theta) \mathrm{d} \theta$.

Next, we investigate the impulsive stochastic chemostat competition model with saturated growth response rates in a polluted environment. The main objective of this paper is to explore the extinction and persistence of a microorganism population and obtain the thresholds of the two chemostat models.

\section{Deterministic system and auxiliary lemmas}

For convenience of discussion, we introduce the following definition and some lemmas.

Definition 2.1 ([50, 51])

(i) The microorganisms $x_{i}(t)$ are said to be extinctive if $\lim _{t \rightarrow+\infty} x_{i}(t)=0(i=1,2)$ a.s.

(ii) The microorganisms $x_{i}(t)$ are said to be persistent if there exist positive constants $\lambda_{i}$ such that $\liminf _{t \rightarrow+\infty} x_{i}(t) \geq \lambda_{i}(i=1,2)$.

(iii) The microorganisms $x_{i}(t)$ are said to be persistent in the mean if there exist positive constants $\lambda_{i}$ such that $\liminf _{t \rightarrow+\infty}\left\langle x_{i}(t)\right\rangle \geq \lambda_{i}(i=1,2)$ a.s. 
The subsystem of systems (1) and (2) is given by

$$
\left\{\begin{array}{l}
\mathrm{d} c(t)=-h c(t) \mathrm{d} t, \quad t \neq n \tau, n \in Z^{+} \\
c\left(n \tau^{+}\right)=c(n \tau)+u, \quad n \in Z^{+} .
\end{array}\right.
$$

Lemma 2.1 [21, 22] System (3) has a unique positive $\tau$-periodic solution $c^{*}(t)$ and, for any solution $c(t)$ of $(3), c(t) \rightarrow c^{*}(t)$ as $t \rightarrow+\infty$. Moreover, $c(t)>c^{*}(t)$ for all $t \geq 0$ if $c(0)>c^{*}(0)$, where

$$
\left\{\begin{array}{l}
c^{*}(t)=\frac{u e^{-h(t-n \tau)}}{1-e^{-h \tau}} \\
c^{*}(0)=\frac{u}{1-e^{-h \tau}}
\end{array}\right.
$$

for $t \in(n \tau,(n+1) \tau]$ and $n \in Z^{+}$.

Lemma 2.2 For any positive solution $\left(S(t), x_{1}(t), x_{2}(t), c(t)\right)$ of deterministic system (1) with initial value $\left(S(0), x_{1}(0), x_{2}(0), c\left(0^{+}\right)\right) \in R_{+}^{4}$, we have

$$
\begin{aligned}
& \limsup _{t \rightarrow+\infty} S(t) \leq S_{0}, \quad \quad \underset{t \rightarrow+\infty}{\limsup x_{1}(t) \leq \delta_{1} S_{0},} \\
& \limsup _{t \rightarrow+\infty} x_{2}(t) \leq \delta_{2} S_{0}, \quad \lim _{t \rightarrow+\infty}\langle c(t)\rangle=\frac{u}{h \tau} \triangleq \bar{c} .
\end{aligned}
$$

Proof From the first three equations of system (1) or (2), we have

$$
\frac{\mathrm{d}\left(S(t)+\frac{1}{\delta_{1}} x_{1}(t)+\frac{1}{\delta_{2}} x_{2}(t)\right)}{\mathrm{d} t} \leq Q S_{0}-Q\left(S(t)+\frac{1}{\delta} x_{1}(t)+\frac{1}{\delta_{2}} x_{2}(t)\right) .
$$

Thus we get

$$
\lim _{t \rightarrow+\infty}\left(S(t)+\frac{1}{\delta_{1}} x_{1}(t)+\frac{1}{\delta_{2}} x_{2}(t)\right) \leq S_{0}
$$

Then

$$
\limsup _{t \rightarrow+\infty} S(t) \leq S_{0}, \quad \limsup _{t \rightarrow+\infty} x_{i}(t) \leq \delta_{i} S_{0}, \quad i=1,2
$$

By Lemma 2.1 we have

$$
\lim _{t \rightarrow+\infty} \frac{1}{t} \int_{0}^{t} c(s) \mathrm{d} s=\lim _{t \rightarrow+\infty} \frac{1}{t} \int_{0}^{t} c^{*}(s) \mathrm{d} s=\frac{1}{\tau} \int_{0}^{\tau} c^{*}(t) \mathrm{d} t=\frac{u}{h \tau} .
$$

The proof of Lemma 2.2 is completed.

Similarly, we can obtain the same results for stochastic system (2), which is used in the following sections.

Define

$$
\mathcal{R}_{1}=\frac{\mu_{1} S_{0}}{a_{1}\left(Q+\frac{r_{1} u}{h \tau}\right)}, \quad \mathcal{R}_{2}=\frac{\mu_{2} S_{0}}{a_{2}\left(Q+\frac{r_{2} u}{h \tau}\right)}
$$


Lemma 2.3 If $\mathcal{R}_{1}<1$ and $\mathcal{R}_{2}<1$, then system (1) has a unique stable 'microorganismextinction' periodic solution $\left(S_{0}, 0,0, c^{*}(t)\right)$, which implies that the two microorganisms go extinct, whereas, if $\mathcal{R}_{1}>1$ and $\mathcal{R}_{2}>1$, then the two microorganisms of system (1) are persistent.

Lemma 2.3 is proved in the Appendix.

Remark 2.1 By Lemma 2.3, two thresholds $\mathcal{R}_{1}$ and $\mathcal{R}_{2}$ decide the persistence and extinction of the microorganisms that are related with the impulsive disturbance force, that is, the larger toxicant pulsed input $u$ or the smaller period of pulsing $\tau$ can lead to the extinction of the microorganisms in the deterministic system (1) without white noise disturbance.

\section{Dynamics of stochastic system}

\subsection{Extinction}

In this section, we investigate the conditions for the extinction of the two microorganisms of system (2) under the stochastic white noise disturbance.

Lemma 3.1 Let $\left(S(t), x_{1}(t), x_{2}(t), c(t)\right)$ be a solution of system (2) with initial value $(S(0)$, $\left.x_{1}(0), x_{2}(0), c(0)\right) \in R_{+}^{4}$. Then

$$
\begin{aligned}
& \lim _{t \rightarrow+\infty} \frac{\int_{0}^{t} \frac{\sigma_{i} S(\theta)}{a_{i}+x_{i}(\theta)} \mathrm{d} B_{i}(\theta)}{t}=0, \\
& \lim _{t \rightarrow+\infty} \frac{\int_{0}^{t} \sigma_{i} S(\theta) \mathrm{d} B_{i}(\theta)}{t}=0, \quad i=1,2 \text {, a.s. }
\end{aligned}
$$

Proof Let $Z(t)=\int_{0}^{t} \frac{\sigma_{i} S(\theta)}{a_{i}+x_{i}(\theta)} \mathrm{d} B_{i}(\theta)$ and $\xi>2$. From Lemma 2.2 and Burkholder-DavisGundy inequality (see [52]) we have

$$
\begin{aligned}
E\left[\sup _{0 \leq \theta \leq t}|Z(\theta)|^{\xi}\right] & \leq C_{\xi} E\left[\int_{0}^{t} \frac{\sigma_{i}^{2} S^{2}(\theta)}{\left(a_{i}+x_{i}(\theta)\right)^{2}} \mathrm{~d} \theta\right]^{\frac{\xi}{2}} \\
& \leq C_{\xi} t^{\frac{\xi}{2}} E\left[\sup _{0 \leq \theta \leq t} \frac{\sigma_{i}^{\xi} S^{\xi}(\theta)}{\left(a_{i}+x_{i}(\theta)\right)^{\xi}}\right] \\
& \leq M_{\xi} C_{\xi} t^{\frac{\xi}{2}},
\end{aligned}
$$

where $M_{\xi}=\left(\frac{S_{0} \sigma_{i}}{a_{i}}\right)^{\xi}$. Let $\varepsilon$ be an arbitrary positive constant. Then we can observe that

$$
\begin{aligned}
\mathbb{P}\left\{\omega: \sup _{k \delta \leq t \leq(k+1) \delta}|Z(t)|^{\xi}>(k \delta)^{1+\varepsilon+\frac{\xi}{2}}\right\} & \leq \frac{E\left(|Z((k+1) \delta)|^{\xi}\right)}{(k \delta)^{1+\varepsilon+\frac{\xi}{2}}} \\
& \leq \frac{M_{\xi} C_{\xi}[(k+1) \delta]^{\frac{\xi}{2}}}{(k \delta)^{1+\varepsilon+\frac{\xi}{2}}} \\
& \leq \frac{2^{\frac{\xi}{2}} M_{\xi} C_{\xi}}{(k \delta)^{1+\varepsilon}} .
\end{aligned}
$$


By the Borel-Cantelli lemma and Doob's martingale inequality (see [52]), for almost all $\omega \in \Omega$, we have that

$$
\sup _{k \delta \leq t \leq(k+1) \delta}|Z(t)|^{\xi} \leq(k \delta)^{1+\varepsilon+\frac{\xi}{2}}
$$

for all but finitely many $k$. Thus, there exists a positive $k_{0}(\omega)$ such that, for almost all $\omega \in \Omega$, (6) holds when $k \geq k_{0}(\omega)$. Hence, if $k \geq k_{0}(\omega)$ and $k \delta \leq t \leq(k+1) \delta$, then, for almost all $\omega \in \Omega$,

$$
\frac{\ln |Z(t)|^{\xi}}{\ln t} \leq \frac{\left(1+\varepsilon+\frac{\xi}{2}\right) \ln (k \delta)}{\ln (k \delta)}=1+\varepsilon+\frac{\xi}{2} .
$$

Thus we have

$$
\limsup _{t \rightarrow+\infty} \frac{\ln |Z(t)|}{\ln t} \leq \frac{1+\varepsilon+\frac{\xi}{2}}{\xi}
$$

Letting $\varepsilon \rightarrow 0$, we obtain

$$
\limsup _{t \rightarrow+\infty} \frac{\ln |Z(t)|}{\ln t} \leq \frac{1}{2}+\frac{1}{\xi}
$$

Then, for an arbitrary small positive constant $\epsilon\left(\epsilon<\frac{1}{2}-\frac{1}{\xi}\right)$, there exist a constant $T(\omega)$ and a set $\Omega_{\epsilon}$ such that $\mathbb{P}\left(\Omega_{\epsilon}\right) \geq 1-\epsilon$ and, for $t \geq T(\omega), \omega \in \Omega_{\epsilon}$,

$$
\ln |Z(t)| \leq\left(\frac{1}{2}+\frac{1}{\xi}+\epsilon\right) \ln t .
$$

Therefore,

$$
\limsup _{t \rightarrow+\infty} \frac{Z(t)}{t} \leq \limsup _{t \rightarrow+\infty} \frac{t^{\frac{1}{2}+\frac{1}{\xi}+\epsilon}}{t}=0 .
$$

Note that

$$
\liminf _{t \rightarrow+\infty} \frac{|Z(t)|}{t} \geq 0
$$

Then we have

$$
\lim _{t \rightarrow+\infty} \frac{|Z(t)|}{t}=0 \quad \text { a.s. }
$$

that is,

$$
\lim _{t \rightarrow+\infty} \frac{Z(t)}{t}=\lim _{t \rightarrow+\infty} \frac{\int_{0}^{t} \frac{\sigma_{i} S(\theta)}{a_{i}+x_{i}(\theta)} \mathrm{d} B_{i}(\theta)}{t}=0 \quad \text { a.s. }
$$

Similarly, we can obtain

$$
\lim _{t \rightarrow+\infty} \frac{\int_{0}^{t} \sigma_{i} S(\theta) \mathrm{d} B_{i}(\theta)}{t}=0, \quad i=1,2, \text { a.s. }
$$

This completes the proof of Lemma 3.1. 
Define

$$
\begin{aligned}
& \mathcal{R}_{1}^{*}=\frac{\mu_{1} S_{0}}{a_{1}\left(Q+\frac{r_{1} u}{h \tau}\right)}-\frac{\sigma_{1}^{2} S_{0}^{2}}{2 a_{1}^{2}\left(Q+\frac{r_{1} u}{h \tau}\right)}=\mathcal{R}_{1}-\frac{\sigma_{1}^{2} S_{0}^{2}}{2 a_{1}^{2}\left(Q+\frac{r_{1} u}{h \tau}\right)}, \\
& \mathcal{R}_{2}^{*}=\frac{\mu_{2} S_{0}}{a_{2}\left(Q+\frac{r_{2} u}{h \tau}\right)}-\frac{\sigma_{2}^{2} S_{0}^{2}}{2 a_{2}^{2}\left(Q+\frac{r_{2} u}{h \tau}\right)}=\mathcal{R}_{2}-\frac{\sigma_{2}^{2} S_{0}^{2}}{2 a_{2}^{2}\left(Q+\frac{r_{2} u}{h \tau}\right)},
\end{aligned}
$$

where $\mathcal{R}_{1}, \mathcal{R}_{2}$ are the thresholds of the deterministic system (1) given in (5).

Theorem 3.1 Let $\left(S(t), x_{1}(t), x_{2}(t), c(t)\right)$ be the solution of system (2) with initial value $\left(S(0), x_{1}(0), x_{2}(0), c(0)\right) \in R_{+}^{4}$. If (i) $\sigma_{i}>\frac{\mu_{i}}{\sqrt{2\left(Q+\frac{r_{i} u}{h \tau}\right)}}$ for $i=1,2$ or (ii) $\mathcal{R}_{i}^{*}<1$ and $\sigma_{i} \leq \sqrt{\frac{a_{i} \mu_{i}}{S_{0}}}$ for $i=1,2$, then the two microorganisms of system (2) go to extinction almost surely, that is, $\lim _{t \rightarrow+\infty} x_{i}(t)=0(i=1,2)$ a.s.; moreover, $\lim _{t \rightarrow+\infty} S(t)=S_{0}$ a.s. and $\lim _{t \rightarrow+\infty} c(t)=c^{*}(t)$ for $t \in(n \tau,(n+1) \tau]$ and $n \in Z^{+}$.

Proof Applying Itô's formula to system (2) leads to

$$
\begin{aligned}
\mathrm{d} \ln x_{i}(t)= & \left(\frac{\mu_{i} S(t)}{a_{i}+x_{i}(t)}-Q-r_{i} c(t)-\frac{\sigma_{i}^{2} S^{2}(t)}{2\left(a_{i}+x_{i}(t)\right)^{2}}\right) \mathrm{d} t \\
& +\frac{\sigma_{i} S(t)}{a_{i}+x_{i}(t)} \mathrm{d} B_{i}(t), \quad i=1,2 .
\end{aligned}
$$

Case (i). Integrating both sides of (7) from 0 to $t$ results in

$$
\begin{aligned}
\ln x_{i}(t) & =-\frac{\sigma_{i}^{2}}{2} \int_{0}^{t}\left(\frac{\mu_{i}}{\sigma_{i}^{2}}-\frac{S(t)}{a_{i}+x_{i}(t)}\right)^{2} \mathrm{~d} t-Q t-r_{i} \int_{0}^{t} c(\theta) \mathrm{d} \theta+\frac{\mu_{i}^{2}}{2 \sigma_{i}^{2}} t+M_{i}(t)+\ln x_{i}(0) \\
& \leq-Q t-r_{i} \int_{0}^{t} c(\theta) \mathrm{d} \theta+\frac{\mu_{i}^{2}}{2 \sigma_{i}^{2}} t+M_{i}(t)+\ln x_{i}(0)
\end{aligned}
$$

where $M_{i}(t)=\int_{0}^{t} \frac{\sigma_{i} S(\theta)}{a_{i}+x_{i}(\theta)} \mathrm{d} B_{i}(\theta), i=1,2$. Dividing both sides of (8) by $t$, we observe that

$$
\frac{\ln x_{i}(t)}{t} \leq-\left(Q+r_{i}\langle c(t)\rangle-\frac{\mu_{i}^{2}}{2 \sigma_{i}^{2}}\right)+\frac{M_{i}(t)}{t}+\frac{\ln x_{i}(0)}{t}
$$

The process $M_{i}(t)(i=1,2)$ is a local continuous martingale with $M_{i}(0)=0$, and from Lemma 3.1 we have

$$
\lim _{t \rightarrow+\infty} \frac{M_{i}(t)}{t}=0, \quad i=1,2 \text {, a.s. }
$$

Since $\sigma_{i}>\frac{\mu_{i}}{\sqrt{2\left(Q+\frac{r_{i} u}{h \tau}\right)}}$ for $i=1,2$, we have $-\left(Q+r_{i}\langle c(t)\rangle-\frac{\mu_{i}^{2}}{2 \sigma_{i}^{2}}\right)<0$.

Taking the limit superior of both sides of (9), we can observe that

$$
\limsup _{t \rightarrow+\infty} \frac{\ln x_{i}(t)}{t} \leq-\left(Q+r_{i}|c(t)\rangle-\frac{\mu_{i}^{2}}{2 \sigma_{i}^{2}}\right)<0 \quad \text { a.s., }
$$

which implies $\lim _{t \rightarrow+\infty} x_{i}(t)=0, i=1,2$, a.s. 
Case (ii). Integrating both sides of (7) from 0 to $t$ and dividing by $t$ lead to

$$
\begin{aligned}
\frac{\ln x_{i}(t)}{t}= & \frac{1}{t} \int_{0}^{t}\left(\frac{\mu_{i} S(\theta)}{a_{i}+x_{i}(\theta)}-Q-r_{i} c(\theta)-\frac{\sigma_{i}^{2} S^{2}(\theta)}{2\left(a_{i}+x_{i}(\theta)\right)^{2}}\right) \mathrm{d} \theta+\frac{M_{i}(t)}{t}+\frac{\ln x_{i}(0)}{t} \\
\leq & \left(\frac{\mu_{i} S_{0}}{a_{i}}-\left(Q+r_{i}\langle c(t)\rangle\right)-\frac{\sigma_{i}^{2} S_{0}^{2}}{2 a_{i}^{2}}\right)+\frac{M_{i}(t)}{t}+\frac{\ln x_{i}(0)}{t} \\
= & \left(Q+r_{i}\langle c(t)\rangle\right)\left(\frac{\mu_{i} S_{0}}{a_{i}\left(Q+r_{i}\langle c(t)\rangle\right)}-\frac{\sigma_{i}^{2} S_{0}^{2}}{2 a_{i}^{2}\left(Q+r_{i}\langle c(t)\rangle\right)}-1\right) \\
& +\frac{M_{i}(t)}{t}+\frac{\ln x_{i}(0)}{t} .
\end{aligned}
$$

Taking the limit superior of both sides of (10) yields

$$
\limsup _{t \rightarrow+\infty} \frac{\ln x_{i}(t)}{t} \leq\left(Q+r_{i} \bar{c}\right)\left(\mathcal{R}_{i}^{*}-1\right)<0 \quad \text { a.s. }
$$

which means $\lim _{t \rightarrow+\infty} x_{i}(t)=0$ a.s.

Without loss of generality, we may assume that $0<x_{i}(t)<\varepsilon_{i}(i=1,2)$ for an arbitrarily small positive quantity $\varepsilon_{i}$ and all $t \geq 0$. By the first equation of system (2) we have

$$
\frac{\mathrm{d} S(t)}{\mathrm{d} t} \geq Q\left(S_{0}-S(t)\right)-\left(\frac{u_{1} \varepsilon_{1}}{\delta_{1} a_{1}}+\frac{u_{2} \varepsilon_{2}}{\delta_{2} a_{2}}+\frac{\sigma_{1} \varepsilon_{1}}{\delta_{1} a_{1}}\left|\dot{B}_{1}(t)\right|+\frac{\sigma_{2} \varepsilon_{2}}{\delta_{2} a_{2}}\left|\dot{B}_{2}(t)\right|\right) S(t) .
$$

As $\varepsilon_{1} \rightarrow 0$ and $\varepsilon_{2} \rightarrow 0$, taking the limit inferior of both sides of (11) gives

$$
\liminf _{t \rightarrow+\infty} S(t) \geq S_{0} \quad \text { a.s. }
$$

By the proof of Lemma 2.2 we have

$$
\lim _{t \rightarrow+\infty} S(t) \leq S_{0}+\varepsilon_{1}+\varepsilon_{2} \quad \text { a.s. }
$$

Then, letting $\varepsilon_{1} \rightarrow 0$ and $\varepsilon_{2} \rightarrow 0$, we have

$$
\limsup _{t \rightarrow+\infty} S(t) \leq S_{0} \quad \text { a.s. }
$$

From (12) and (13) we have

$$
\lim _{t \rightarrow+\infty} S(t)=S_{0} \quad \text { a.s. }
$$

From (3) and Lemma 2.1 we can observe that

$$
\lim _{t \rightarrow+\infty} c(t)=c^{*}(t)
$$

for $t \in(n \tau,(n+1) \tau]$ and $n \in Z^{+}$.

Remark 3.1 Theorem 3.1 shows that the two microorganisms will die out if the white noise disturbance is large or $\mathcal{R}_{i}^{*}<1$ and the white noise disturbance is not too large. Note 
that the expression of $\mathcal{R}_{i}^{*}$ is a difference compared with two thresholds of system (1), $\mathcal{R}_{i}$. This implies that the conditions for $x_{i}(t)$ to go to extinction in the deterministic system (1) are stronger than in the corresponding stochastic model (2).

\subsection{Persistence in mean}

Theorem 3.2 Let $\left(S(t), x_{1}(t), x_{2}(t), c(t)\right)$ be a solution of system (2) with initial value $\left(S(0), x_{1}(0), x_{2}(0), c(0)\right) \in R_{+}^{4}$.

(i) If $\mathcal{R}_{1}^{*}>1, \mathcal{R}_{2}^{*}<1$, and $\sigma_{2} \leq \sqrt{\frac{a_{2} \mu_{2}}{S_{0}}}$, then the microorganism $x_{2}$ dies out, and the microorganism $x_{1}$ is persistent in mean; moreover, $x_{1}$ satisfies

$$
\liminf _{t \rightarrow+\infty}\left\langle x_{1}(t)\right\rangle \geq \frac{a_{1} \delta_{1} Q\left(Q+r_{1} \bar{c}\right)}{\left(\mu_{1}+\delta_{1} Q\right)\left(Q+r_{1} c^{*}(0)\right)}\left(\mathcal{R}_{1}^{*}-1\right) \quad \text { a.s. }
$$

(ii) If $\mathcal{R}_{2}^{*}>1, \mathcal{R}_{1}^{*}<1$, and $\sigma_{1} \leq \sqrt{\frac{a_{1} \mu_{1}}{S_{0}}}$, then the microorganism $x_{1}$ dies out, and the microorganism $x_{2}$ is persistent in mean; moreover, $x_{2}$ satisfies

$$
\liminf _{t \rightarrow+\infty}\left\langle x_{2}(t)\right\rangle \geq \frac{a_{2} \delta_{2} Q\left(Q+r_{2} \bar{c}\right)}{\left(\mu_{2}+\delta_{2} Q\right)\left(Q+r_{2} c^{*}(0)\right)}\left(\mathcal{R}_{2}^{*}-1\right) \quad \text { a.s. }
$$

(iii) If $\mathcal{R}_{1}^{*}>1$ and $\mathcal{R}_{2}^{*}>1$, then the two microorganisms $x_{1}$ and $x_{2}$ are persistent in mean; moreover, $x_{1}$ and $x_{2}$ satisfy

$$
\liminf _{t \rightarrow+\infty}\left\langle x_{1}(t)+x_{2}(t)\right\rangle \geq \frac{1}{\Delta_{\max }} \sum_{i=1}^{2} a_{i}\left(Q+r_{i} \bar{c}\right)\left(\mathcal{R}_{i}^{*}-1\right) \quad \text { a.s. }
$$

where

$$
\Delta_{\max }=\max \left\{\left(Q+r_{1} c^{*}(0)\right)\left(\frac{\mu_{1}+\mu_{2}}{\delta_{1} Q}+1\right),\left(Q+r_{2} c^{*}(0)\right)\left(\frac{\mu_{1}+\mu_{2}}{\delta_{2} Q}+1\right)\right\} .
$$

Proof Case (i). By Theorem 3.1, since $\mathcal{R}_{2}^{*}<1$ and $\sigma_{2} \leq \sqrt{\frac{a_{2} \mu_{2}}{S_{0}}}$, we have $\lim _{t \rightarrow+\infty} x_{2}(t)=0$ a.s. Since $\mathcal{R}_{1}^{*}>1$, we have that, for $\varepsilon$ small enough such that $0<x_{2}(t)<\varepsilon$ for all $t$ large enough,

$$
\frac{\mu_{1}\left(S_{0}-\left(\frac{Q+r_{2} c^{*}(0)}{\delta_{3} Q} \varepsilon\right)\right)}{a_{1}(Q+r \bar{c})}-\frac{\sigma_{1}^{2} S_{0}^{2}}{2 a_{1}^{2}\left(Q+r_{1} \bar{c}\right)}>1 \quad \text { a.s. }
$$

Integrating both sides of system (2) from 0 to $t$ and dividing by $t$ yield

$$
\begin{aligned}
\Theta(t) & \triangleq \frac{S(t)-S(0)}{t}+\frac{1}{\delta_{1}} \frac{x_{1}(t)-x_{1}(0)}{t}+\frac{1}{\delta_{2}} \frac{x_{2}(t)-x_{2}(0)}{t} \\
& \geq Q S_{0}-Q\langle S(t)\rangle-\left(\frac{Q+r_{1} c^{*}(0)}{\delta_{1}}\right)\left\langle x_{1}(t)\right\rangle-\left(\frac{Q+r_{2} c^{*}(0)}{\delta_{2}}\right)\left\langle x_{2}(t)\right\rangle \\
& \geq Q S_{0}-Q\langle S(t)\rangle-\left(\frac{Q+r_{1} c^{*}(0)}{\delta_{1}}\right)\left\langle x_{1}(t)\right\rangle-\left(\frac{Q+r_{2} c^{*}(0)}{\delta_{2}}\right) \varepsilon .
\end{aligned}
$$

Then we get

$$
\langle S(t)\rangle \geq\left(S_{0}-\left(\frac{Q+r_{2} c^{*}(0)}{\delta_{2} Q}\right) \varepsilon\right)-\left(\frac{Q+r_{1} c^{*}(0)}{\delta_{1} Q}\right)\left\langle x_{1}(t)\right\rangle-\frac{\Theta(t)}{Q} .
$$


Applying Itô's formula to system (2) leads to

$$
\begin{aligned}
& \mathrm{d}\left(a_{1} \ln x_{1}(t)+x_{1}(t)\right) \\
& \quad=\left(\mu_{1} S(t)-a_{1}\left(Q+r_{1} c(t)\right)-\left(Q+r_{1} c(t)\right) x_{1}(t)-\frac{a_{1} \sigma_{1}^{2} S^{2}(t)}{2\left(a_{1}+x_{1}(t)\right)^{2}}\right) \mathrm{d} t+\sigma_{1} S(t) \mathrm{d} B_{1}(t) \\
& \quad \geq\left(\mu_{1} S(t)-a_{1}\left(Q+r_{1} c(t)\right)-\left(Q+r_{1} c^{*}(0)\right) x_{1}(t)-\frac{\sigma_{1}^{2} S_{0}^{2}}{2 a_{1}}\right) \mathrm{d} t+\sigma_{1} S(t) \mathrm{d} B_{1}(t) .
\end{aligned}
$$

Integrating on both sides of (15) from 0 to $t$ and dividing by $t$ yield

$$
\begin{aligned}
& \frac{a_{1}\left(\ln x_{1}(t)-\ln x_{1}(0)\right)}{t}+\frac{x_{1}(t)-x_{1}(0)}{t} \\
& \geq \mu_{1}\langle S(t)\rangle-a_{1}\left(Q+r_{1}\langle c(t)\rangle\right)-\left(Q+r_{1} c^{*}(0)\right)\left\langle x_{1}(t)\right\rangle-\frac{\sigma_{1}^{2} S_{0}^{2}}{2 a_{1}}+\frac{M_{1}(t)}{t} \\
& \geq \mu_{1}\left(S_{0}-\left(\frac{Q+r_{2} c^{*}(0)}{\delta_{2} Q}\right) \varepsilon\right)-a_{1}\left(Q+r_{1}\langle c(t)\rangle\right)-\frac{\sigma_{1}^{2} S_{0}^{2}}{2 a_{1}} \\
& \quad-\left(\frac{\mu_{1}\left(Q+r_{1} c^{*}(0)\right)}{\delta_{1} Q}+\left(Q+r_{1} c^{*}(0)\right)\right)\left\langle x_{1}(t)\right\rangle-\frac{\mu_{1} \Theta(t)}{Q}+\frac{M_{1}(t)}{t} \\
& =a_{1}\left(Q+r_{1}\langle c(t)\rangle\right)\left(\frac{\mu_{1}\left(S_{0}-\frac{Q+r_{2} c^{*}(0)}{\delta_{2} Q} \varepsilon\right)}{a_{1}\left(Q+r_{1}\langle c(t)\rangle\right)}-\frac{\sigma_{1}^{2} S_{0}^{2}}{2 a_{1}\left(Q+r_{1}\langle c(t)\rangle\right)}-1\right) \\
& \quad-\left(\frac{\mu_{1}\left(Q+r_{1} c^{*}(0)\right)}{\delta_{1} Q}+\left(Q+r_{1} c^{*}(0)\right)\right)\left\langle x_{1}(t)\right\rangle-\frac{\mu_{1} \Theta(t)}{Q}+\frac{M_{1}(t)}{t}
\end{aligned}
$$

where $M_{1}(t)=\int_{0}^{t} \sigma_{1} S(\theta) \mathrm{d} B_{1}(\theta)$. Inequality (16) can be rewritten as

$$
\begin{aligned}
\left\langle x_{1}(t)\right\rangle \geq & \frac{1}{\Delta}\left[a_{1}\left(Q+r_{1}\langle c(t)\rangle\right)\left(\frac{\mu_{1}\left(S_{0}-\frac{Q+r_{2} c^{*}(0)}{\delta_{2} Q} \varepsilon\right)}{a_{1}\left(Q+r_{1}\langle c(t)\rangle\right)}-\frac{\sigma_{1}^{2} S_{0}^{2}}{2 a_{1}^{2}\left(Q+r_{1}\langle c(t)\rangle\right)}-1\right)\right. \\
& \left.-\frac{\mu_{1} \Theta(t)}{Q}+\frac{M_{1}(t)}{t}-\left(\frac{a_{1}\left(\ln x_{1}(t)-\ln x_{1}(0)\right)}{t}+\frac{x_{1}(t)-x_{1}(0)}{t}\right)\right] \\
\geq & \left\{\begin{array}{c}
\frac{1}{\Delta}\left[a_{1}\left(Q+r_{1}\langle c(t)\rangle\right)\left(\frac{\mu_{1}\left(S_{0}-\frac{Q+r_{2} c^{*}(0)}{\delta_{2} Q} \varepsilon\right)}{a_{1}\left(Q+r_{1}\langle c(t)\rangle\right)}-\frac{\sigma_{1}^{2} S_{0}^{2}}{2 a_{1}^{2}\left(Q+r_{1}\langle c(t)\rangle\right)}-1\right)\right. \\
\left.-\frac{\mu_{1} \Theta(t)}{Q}+\frac{M_{1}(t)}{t}+\frac{a_{1} \ln x_{1}(0)}{t}-\frac{x_{1}(t)-x_{1}(0)}{t}\right], \quad 0<x_{1}(t)<1 ; \\
\frac{1}{\Delta}\left[a_{1}\left(Q+r_{1}\langle c(t)\rangle\right)\left(\frac{\mu_{1}\left(S_{0}-\frac{Q+r_{2} c^{*}(0)}{\delta_{2} Q} \varepsilon\right)}{a_{1}\left(Q+r_{1}\langle c(t)\rangle\right)}-\frac{\sigma_{1}^{2} S_{0}^{2}}{2 a_{1}^{2}\left(Q+r_{1}\langle c(t)\rangle\right)}-1\right)\right. \\
\left.-\frac{\mu_{1} \Theta(t)}{Q}+\frac{M_{1}(t)}{t}-\frac{a_{1}\left(\ln x_{1}(t)-\ln x_{1}(0)\right)}{t}-\frac{x_{1}(t)-x_{1}(0)}{t}\right], \quad 1 \leq x_{1}(t),
\end{array}\right.
\end{aligned}
$$

where $\Delta=\frac{\left(Q+r_{1} c^{*}(0)\right)\left(\mu_{1}+\delta_{1} Q\right)}{\delta_{1} Q}$.

By Lemma 3.1 we have $\lim _{t \rightarrow+\infty} \frac{M_{1}(t)}{t}=0$ a.s. According to Lemma 2.2, we can find that $x_{1}(t) \leq \delta_{1} S_{0}$. Thus we have $\lim _{t \rightarrow+\infty} \frac{x_{1}(t)}{t}=0$ and $\lim _{t \rightarrow+\infty} \frac{\ln x_{1}(t)}{t}=0$ a.s. as $x_{1}(t) \geq 1$ and $\lim _{t \rightarrow+\infty} \Theta(t)=0$ a.s. Taking the limit inferior of both sides of (17) results in

$$
\begin{aligned}
\liminf _{t \rightarrow+\infty}\left|x_{1}(t)\right\rangle & \geq \frac{a_{1}\left(Q+r_{1} \bar{c}\right)}{\Delta}\left[\frac{\mu_{1} S_{0}}{a_{1}\left(Q+r_{1} \bar{c}\right)}-\frac{\sigma_{1}^{2} S_{0}^{2}}{2 a_{1}^{2}\left(Q+r_{1} \bar{c}\right)}-1\right] \\
& =\frac{a_{1} \delta_{1} Q\left(Q+r_{1} \bar{c}\right)}{\left(\mu_{1}+\delta_{1} Q\right)\left(Q+r_{1} c^{*}(0)\right)}\left(\mathcal{R}_{1}^{*}-1\right)>0 .
\end{aligned}
$$

Similarly, we can prove Case (ii), and we omit it here. 
Case (iii). Note that

$$
\langle S(t)\rangle=S_{0}-\frac{Q+r_{2}\langle c(t)\rangle}{\delta_{2} Q}\left\langle x_{2}(t)\right\rangle-\frac{Q+r_{1}\langle c(t)\rangle}{\delta_{1} Q}\left\langle x_{1}(t)\right\rangle-\frac{\Theta(t)}{Q} .
$$

Define

$$
V(t)=\ln \left[x_{1}^{a_{1}}(t) x_{2}^{a_{2}}(t)\right]+\left[x_{1}(t)+x_{2}(t)\right]
$$

Note that $V(t)$ is bounded. Then we have

$$
\begin{aligned}
D^{+} V(t)= & {\left[\left(\mu_{1}+\mu_{2}\right) S(t)-\sum_{i=1}^{2}\left(Q+r_{i} c(t)\right)\left(a_{i}+x_{i}(t)\right)-\sum_{i=1}^{2} \frac{a_{i} \sigma_{i}^{2} S^{2}(t)}{2\left(a_{i}+x_{i}(t)\right)^{2}}\right] \mathrm{d} t } \\
& +\sum_{i=1}^{2} \sigma_{i} S(t) \mathrm{d} B_{i}(t) \\
\geq & {\left[\left(\mu_{1}+\mu_{2}\right) S(t)-\sum_{i=1}^{2} a_{i}\left(Q+r_{i} c(t)\right)-\sum_{i=1}^{2} x_{i}(t)\left(Q+r_{i} c^{*}(0)\right)-\sum_{i=1}^{2} \frac{\sigma_{i}^{2} S_{0}^{2}}{2 a_{i}}\right] \mathrm{d} t } \\
& +\sum_{i=1}^{2} \sigma_{i} S(t) \mathrm{d} B_{i}(t) .
\end{aligned}
$$

Integrating both sides of (19) from 0 to $t$ and dividing by $t$ yield

$$
\begin{aligned}
\frac{V(t)}{t}- & \frac{V(0)}{t} \\
\geq & \left(\mu_{1}+\mu_{2}\right)\langle S(t)\rangle-\sum_{i=1}^{2} a_{i}\left(Q+r_{i}|c(t)\rangle\right)-\sum_{i=1}^{2}\left\langle x_{i}(t)\right\rangle\left(Q+r_{i} c^{*}(0)\right) \\
& -\sum_{i=1}^{2} \frac{\sigma_{i}^{2} S_{0}^{2}(t)}{2 a_{i}}+\sum_{i=1}^{2} \frac{M_{i}}{t} \\
= & \left(\mu_{1}+\mu_{2}\right) S_{0}-\sum_{i=1}^{2}\left(Q+r_{i}|c(t)|\right) a_{i}-\sum_{i=1}^{2} \frac{\sigma_{i}^{2} S_{0}}{2 a_{i}} \\
& -\sum_{i=1}^{2}\left[\frac{\left(\mu_{1}+\mu_{2}\right)\left(Q+r_{i}\langle c(t)\rangle\right)}{\delta_{i} Q}+\left(Q+r_{i} c^{*}(0)\right)\right]\left\langle x_{i}(t)\right\rangle \\
& -\frac{\mu_{1}+\mu_{2}}{Q} \Theta(t)+\sum_{i=1}^{2} \frac{M_{i}}{t} \\
\geq & \left(\mu_{1}+\mu_{2}\right) S_{0}-\sum_{i=1}^{2}\left(Q+r_{i}\langle c(t)\rangle\right) a_{i}-\sum_{i=1}^{2} \frac{\sigma_{i}^{2} S_{0}}{2 a_{i}}-\Delta_{\max }\left[\left\langle x_{1}(t)\right\rangle+\left\langle x_{2}(t)\right\rangle\right] \\
& -\frac{\mu_{1}+\mu_{2}}{Q} \Theta(t)+\sum_{i=1}^{2} \frac{M_{i}}{t},
\end{aligned}
$$


where $M_{i}(t)=\int_{0}^{t} \sigma_{i} S(\theta) \mathrm{d} B_{i}(\theta)$. Inequality (20) can be rewritten as

$$
\begin{aligned}
\left\langle x_{1}(t)\right\rangle+\left\langle x_{2}(t)\right\rangle \geq & \frac{1}{\Delta_{\max }}\left[\left(\mu_{1}+\mu_{2}\right) S_{0}-\sum_{i=1}^{2}\left(Q+r_{i}|c(t)\rangle\right) a_{i}-\sum_{i=1}^{2} \frac{\sigma_{i}^{2} S_{0}}{2 a_{i}}\right. \\
& \left.-\frac{\mu_{1}+\mu_{2}}{Q} \Theta(t)-\frac{V(t)}{t}+\frac{V(0)}{t}+\sum_{i=1}^{2} \frac{M_{i}}{t}\right] .
\end{aligned}
$$

Since $0<S \leq S_{0}$, we have

$$
\limsup _{t \rightarrow+\infty} \frac{\left\langle M_{i}(t), M_{i}(t)\right\rangle_{t}}{t} \leq \sigma^{2} S_{0}^{2}<\infty \quad \text { a.s. }
$$

By Lemma 3.1 we observe that $\lim _{t \rightarrow+\infty} \frac{M_{i}(t)}{t}=0$ a.s. for $i=1,2$. According to Lemma 2.2, we have $\lim _{t \rightarrow+\infty} \Theta(t)=0$ and $\lim _{t \rightarrow+\infty} \frac{V(t)}{t}=0$.

Taking the limit inferior of both sides of (21) yields

$$
\liminf _{t \rightarrow+\infty}\left\langle x_{1}(t)+x_{2}(t)\right\rangle \geq \frac{1}{\Delta_{\max }} \sum_{i=1}^{2} a_{i}\left(Q+r_{i} \bar{c}\right)\left(\mathcal{R}_{i}^{*}-1\right)>0 \quad \text { a.s. }
$$

This completes the proof of Theorem 3.2.

Remark 3.2 Theorem 3.2 shows that the two microorganisms will be persistent if the white noise disturbances are small enough such that $\mathcal{R}_{i}^{*}>1$; conversely, if the white noise disturbances are large enough, then the two microorganisms will go to extinction. This implies that the stochastic disturbance may cause the populations to die out.

\section{Conclusion and simulations}

In this paper, we investigate the dynamics of an impulsive stochastic competition chemostat model with saturated growth rate in a polluted environment. We obtain sufficient conditions for extinction and persistence of both deterministic and stochastic systems. From the expressions of the thresholds of the stochastic system (2) we can observe that $\mathcal{R}_{i}^{*}<\mathcal{R}_{i}, i=1,2$, which means that the conditions for those two microorganisms to die out in the deterministic model (1) are stronger than those in the corresponding stochastic system (2). This implies that a persistent deterministic system may become extinct in the case of white noise stochastic disturbance.

On one hand, [44-49] investigated the asymptotic stability of some impulsive stochastic differential systems and obtained many good results. On the other hand, [53, 54] investigated qualitative properties for persistence and extinction of one-dimensional impulsive stochastic single-species population models. Based on the works $[53,54]$, we consider the qualitative analysis of the high-dimensional impulsive stochastic multi-species population model, which leads to a more complex and difficult stochastic analysis. Moreover, we use impulsive stochastic inequality technique to discuss the question according to three different cases. The main aim of the paper is to study the stochastic dynamics of the highdimensional impulsive stochastic chemostat model and find the threshold between persistence and extinction of the microorganisms. In a sense, we improve and develop the theoretical method in $[53,54]$. 
(a)

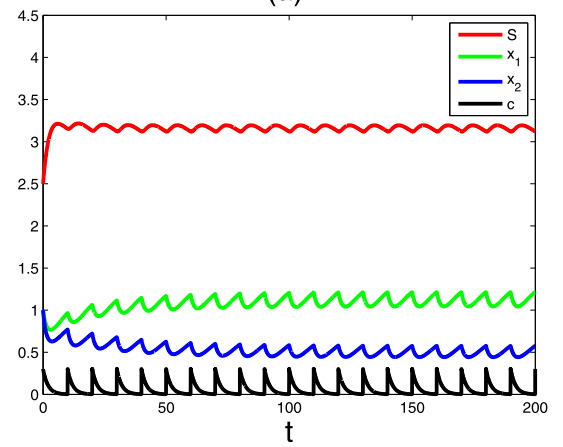

(c)

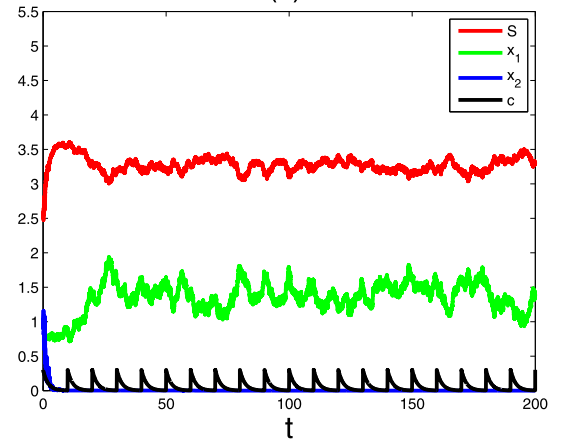

(b)

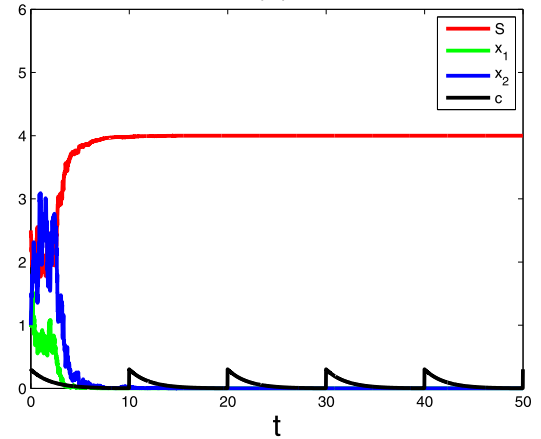

(d)

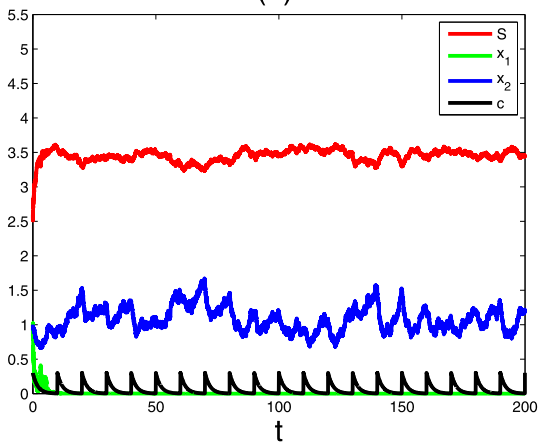

(e)

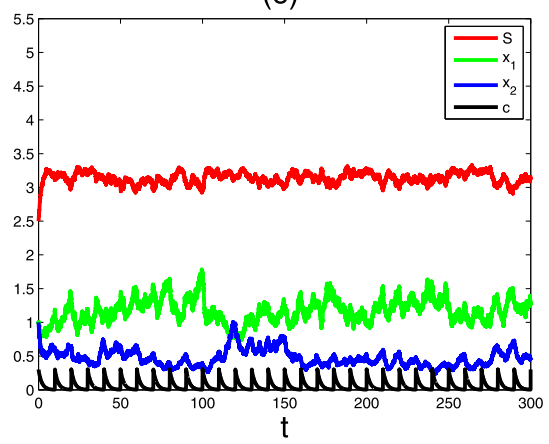

Figure 1 Computer simulation of the paths $S(t), x_{1}(t), x_{2}(t), c(t)$ for the deterministic chemostat model (1) and the stochastic chemostat model (2) with parameters $S_{0}=4, Q=0.5, r_{1}=0.5, r_{2}=0.9, \delta_{1}=2$, $\delta_{2}=2.2, a_{1}=15, a_{2}=7.5, \mu_{1}=2.7, \mu_{2}=1.4, h=0.5, u=0.3, \tau=10$ and the initial values $S(0)=2.5$, $\boldsymbol{x}_{\mathbf{1}}(\mathbf{0})=\mathbf{1}, \boldsymbol{x}_{\mathbf{2}}(\mathbf{0})=\mathbf{1}, \boldsymbol{c}(\mathbf{0})=\mathbf{0 . 3}$. (a) Time series for $S(t), X_{1}(t), X_{2}(t), c(t)$ with parameters $\sigma_{1}=0, \sigma_{2}=0$. (b) Time series for $S(t), X_{1}(t), X_{2}(t), c(t)$ with parameters $\sigma_{1}=2.4, \sigma_{2}=1$.2. (c) Time series for $S(t), X_{1}(t), X_{2}(t), c(t)$ with parameters $\sigma_{1}=0.2, \sigma_{2}=1.2$. (d) Time series for $S(t), X_{1}(t), X_{2}(t), c(t)$ with parameters $\sigma_{1}=2.4, \sigma_{2}=0.1$. (e) Time series for $S(t), X_{1}(t), X_{2}(t), c(t)$ with parameters $\sigma_{1}=0.2, \sigma_{2}=0.1$.

Next, we employ the Euler method to simulate the dynamics of the deterministic and stochastic systems to support our theoretical results. We choose some parameters in systems (1) and (2) as follows: $S_{0}=4, Q=0.5, r_{1}=0.5, r_{2}=0.9, \delta_{1}=2, \delta_{2}=2.2, a_{1}=15$, $a_{2}=7.5, \mu_{1}=2.7, \mu_{2}=1.4, h=0.5, u=0.3, \tau=10$, and the initial values are $S(0)=2.5$, $x_{1}(0)=1, x_{2}(0)=1, c(0)=0.3$.

In Figure 1, we can see that
(a) $\sigma_{1}=0$,
$\sigma_{2}=0$,
$\mathcal{R}_{1}=1.3585>1$,
$\mathcal{R}_{2}=1.3478>1 ;$
(b) $\sigma_{1}=2.4$,
$\sigma_{2}=1.2$,
$\mathcal{R}_{1}^{*}=0.9721<1$,
$\mathcal{R}_{2}^{*}=0.9781<1$. 
This shows that the persistent two microorganisms of a deterministic system (see Figure 1(a)) can become extinct under the white noise stochastic disturbance (see Figure 1(b)), and thus the simulation is consistent with the theoretical results of Lemma 2.3 and Theorem 3.1. When $\mathcal{R}_{i}^{*}=\mathcal{R}_{i}-\frac{\sigma_{i}^{2} S_{0}^{2}}{2 a_{i}^{2}\left(Q+r_{i} \bar{c}\right)}<1<\mathcal{R}_{i}$, a persistent deterministic system goes to extinction due to the white noise disturbance.

Next, we choose $\sigma_{1}$ and $\sigma_{2}$ with different values. When $\sigma_{1}$ is small and $\sigma_{2}$ is large $\left(\sigma_{1}=0.2\right.$, $\sigma_{2}=1.2$ ), here $\mathcal{R}_{1}^{*}=1.3558>1$ and $\mathcal{R}_{2}^{*}=0.9781<1$. Thus, the microorganism $x_{2}$ goes to extinction, and the microorganism $x_{1}$ is persistent (see Figure $1(\mathrm{c})$ ). Conversely, when $\sigma_{1}$ is large and $\sigma_{2}$ is small $\left(\sigma_{1}=2.4, \sigma_{2}=0.1\right)$, here $\mathcal{R}_{1}^{*}=0.9721<1, \mathcal{R}_{2}^{*}=1.3452>1$. Figure $1(\mathrm{~d})$ shows that the microorganism $x_{1}$ goes to extinction and the microorganism $x_{2}$ is persistent. Moreover, for small noise intensities, $\sigma_{1}=0.2$ and $\sigma_{2}=0.1$, both microorganisms are persistent (see Figure 1(e)). This supports our theoretical results in Theorem 3.2, and we observe that the white noise has unfavorable effects on the persistence of microorganisms.

Figure 2(a) shows that a greater impulsive toxicant input can lead to the extinction of the two microorganisms, whereas the microorganism populations can be persistent in the smaller impulsive toxicant input environment (see Figure 2(b) and Figure 2(c)). This supports our theoretical results in Theorems 3.1 and 3.2, and we observe that the impulsive toxicant input has unfavorable effects on the persistence of microorganisms.

From the theoretical analysis and simulations we can find that if the intensity of the white noise or impulsive input is small, then the microorganisms can still be persistent just as in the deterministic system, whereas for the large intensity of the white noise or impulsive input, microorganisms may become extinct. Therefore, noises and impulsive

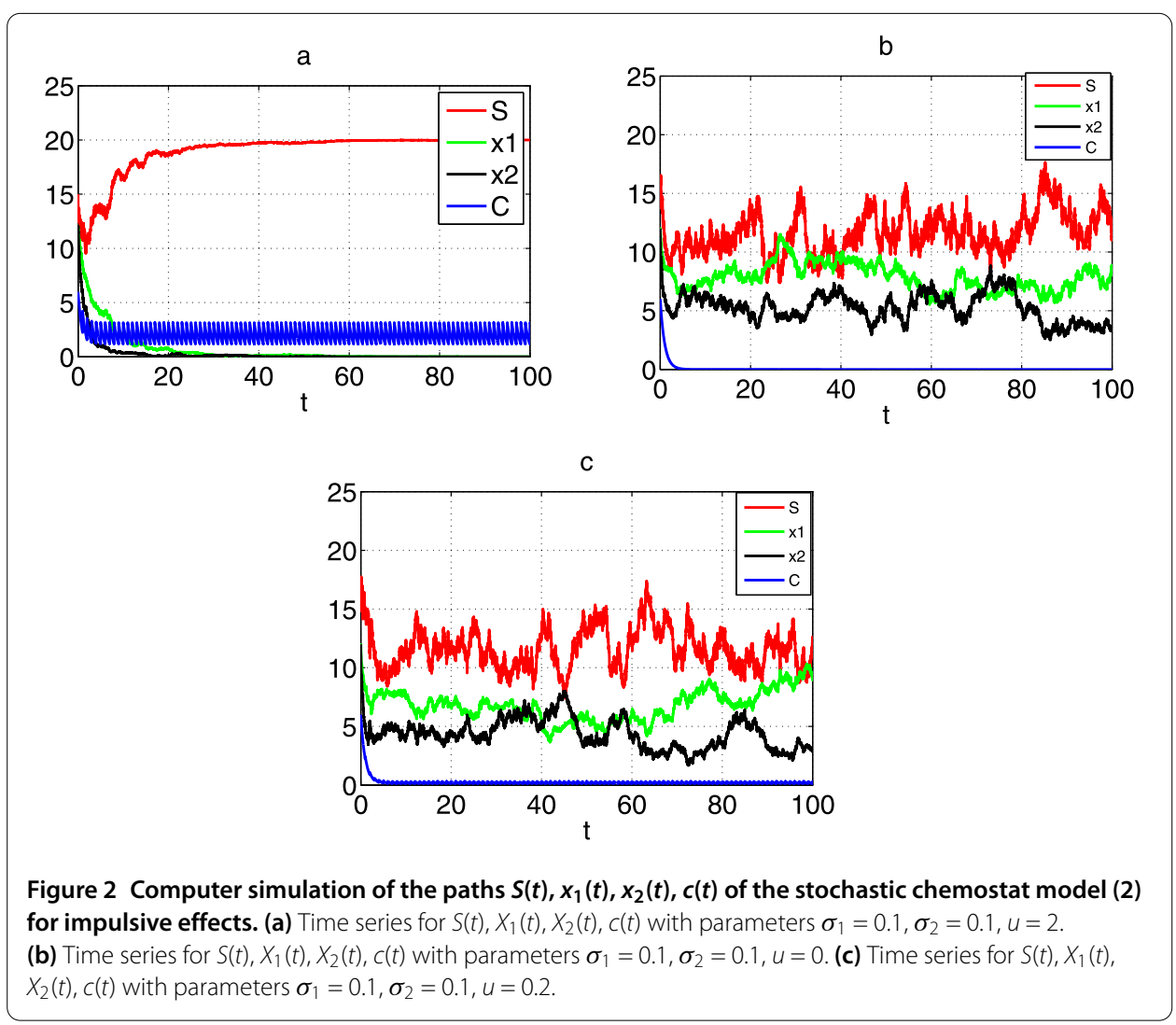


effects go against the survival of microorganisms. The first three equations of system (2) can also be considered as a nonautonomous and nonimpulsive periodic system with periodic coefficient $c(t)$ (with period $\tau$ ). Moreover, the extinction and persistence of two microorganisms are discussed in three cases. The theoretical method can also be used to explore the thresholds of some high-dimensional impulsive stochastic differential systems and some nonautonomous periodic systems.

Some problems in this direction deserve further investigation. It is interesting to study other kinds of high-dimensional impulsive stochastic Lotka-Volterra systems, such as predator-prey system and cooperation system, or introduce a Markov process or Lévy jumps into the impulsive stochastic environment. This our future research work should continue to be concerned about.

\section{Appendix}

The proof of Lemma 2.3 .

Proof By Lemma 2.1 there is a unique 'microorganism-extinction' periodic solution $\left(S_{0}, 0,0, c^{*}(t)\right)$ in system $(1)$. The local stability of the periodic solution $\left(S_{0}, 0,0, c^{*}(t)\right)$ may be determined by considering the behavior of small amplitude perturbations of the solution. Let $S(t)=S_{0}+\phi(t), x_{1}(t)=v_{1}(t), x_{2}(t)=v_{2}(t), c(t)=c^{*}(t)+w(t)$. Then we have

$$
\left(\begin{array}{l}
\phi(t) \\
v_{1}(t) \\
v_{2}(t) \\
w(t)
\end{array}\right)=\Phi(t)\left(\begin{array}{l}
\phi(0) \\
v_{1}(0) \\
v_{2}(0) \\
w(0)
\end{array}\right), \quad 0 \leq t \leq \tau
$$

where $\Phi(t)$ satisfies

$$
\frac{\mathrm{d} \Phi(t)}{\mathrm{d} t}=\left(\begin{array}{cccc}
-Q & * & * & 0 \\
0 & \frac{\mu_{1} S_{0}}{a_{1}}-Q-r_{1} c^{*}(t) & 0 & 0 \\
0 & 0 & \frac{\mu_{2} S_{0}}{a_{2}}-Q-r_{2} c^{*}(t) & 0 \\
0 & 0 & 0 & -h
\end{array}\right) \Phi(t)
$$

$\Phi(0)=E_{4 \times 4}$ is the identity matrix, and the fundamental solution matrix is

$$
\Phi(t)=\left(\begin{array}{cccc}
\exp (-Q \tau) & * & * & 0 \\
0 & \exp \left(\int_{0}^{\tau} \frac{\mu_{1} S_{0}}{a_{1}}-Q-r_{1} c^{*}(t) \mathrm{d} t\right) & 0 & 0 \\
0 & 0 & \exp \left(\int_{0}^{\tau} \frac{\mu_{2} S_{0}}{a_{2}}-Q-r_{2} c^{*}(t) \mathrm{d} t\right) & 0 \\
0 & 0 & 0 & \exp (-h \tau)
\end{array}\right)
$$

There is no need to calculate the exact form of $(*)$ because it is not required in the analysis that follows. The linearization of the impulsive equations of (1) becomes

$$
\left(\begin{array}{l}
\phi\left(n \tau^{+}\right) \\
v_{1}\left(n \tau^{+}\right) \\
v_{2}\left(n \tau^{+}\right) \\
w\left(n \tau^{+}\right)
\end{array}\right)=\left(\begin{array}{llll}
1 & 0 & 0 & 0 \\
0 & 1 & 0 & 0 \\
0 & 0 & 1 & 0 \\
0 & 0 & 0 & 1
\end{array}\right)\left(\begin{array}{l}
\phi(n \tau) \\
v_{1}(n \tau) \\
v_{2}(n \tau) \\
w(n \tau)
\end{array}\right)=E_{4 \times 4}\left(\begin{array}{c}
\phi(n \tau) \\
v_{1}(n \tau) \\
v_{2}(n \tau) \\
w(n \tau)
\end{array}\right) .
$$


The stability of the periodic solution $\left(S_{0}, 0,0, c^{*}(t)\right)$ is determined by the eigenvalues of $M=E_{4 \times 4} \Phi(t)$, that is,

$$
M=\left(\begin{array}{cccc}
\exp (-Q \tau) & \exp \left(\int_{0}^{\tau} \frac{\mu_{1} S_{0}}{a_{1}}-Q-r_{1} c^{*}(t) \mathrm{d} t\right) & * & 0 \\
0 & 0 & 0 & 0 \\
0 & 0 & \exp \left(\int_{0}^{\tau} \frac{\mu_{2} S_{0}}{a_{2}}-Q-r_{2} c^{*}(t) \mathrm{d} t\right) & 0 \\
0 & \exp (-h \tau)
\end{array}\right)
$$

and there is no need to calculate the exact form of $(*)$. The eigenvalues of the upper triangular matrix $M$ are

$$
\begin{aligned}
& \lambda_{1}=\mathrm{e}^{-Q \tau}<1, \quad \lambda_{2}=\mathrm{e}^{\int_{0}^{\tau} \frac{\mu_{1} S_{0}}{a_{1}}-Q-r_{1} c^{*}(t) \mathrm{d} t}, \\
& \lambda_{3}=\mathrm{e}^{\int_{0}^{\tau} \frac{\mu_{2} S_{0}}{a_{2}}-Q-r_{2} c^{*}(t) \mathrm{d} t}, \quad \lambda_{4}=\mathrm{e}^{-h \tau}<1 .
\end{aligned}
$$

According to Floquet theory of impulsive equations, $\left(S_{0}, 0,0, c^{*}(t)\right)$ is stable if $\lambda_{2}<1$ and $\lambda_{3}<1$, that is, $\mathcal{R}_{1}<1$ and $\mathcal{R}_{2}<1$.

Now we prove that system (1) is persistent if $\mathcal{R}_{1}>1$ and $\mathcal{R}_{2}>1$.

For the equation $S(t)+\frac{1}{\delta_{1}} x_{1}(t)+\frac{1}{\delta_{2}} x_{2}(t)$, integrating it from 0 to $t$ and dividing by $t$, for $t$ large enough, we have

$$
\begin{aligned}
\epsilon(t) & \triangleq \frac{S(t)-S(0)}{t}+\frac{1}{\delta_{1}} \frac{x_{1}(t)-x_{1}(0)}{t}+\frac{1}{\delta_{2}} \frac{x_{2}(t)-x_{2}(0)}{t} \\
& \geq Q S_{0}-Q\langle S(t)\rangle-\frac{Q+r_{1} c^{*}(0)}{\delta_{1}}\left\langle x_{1}(t)\right\rangle-\frac{Q+r_{2} c^{*}(0)}{\delta_{2}}\left\langle x_{2}(t)\right\rangle .
\end{aligned}
$$

Then we have

$$
\langle S(t)\rangle \geq S_{0}-\frac{Q+r_{1} c^{*}(0)}{\delta_{1} Q}\left\langle x_{1}(t)\right\rangle-\frac{Q+r_{2} c^{*}(0)}{\delta_{2} Q}\left\langle x_{2}(t)\right\rangle-\frac{\epsilon(t)}{Q} .
$$

Define $V(t)=a_{1} \ln x_{1}(t)+a_{2} \ln x_{2}(t)+x_{1}(t)+x_{2}(t)$, which is a bounded function. Then we get

$$
\begin{aligned}
D^{+} V(t)= & \mu_{1} S(t)-a_{1}\left(Q+r_{1} c(t)\right)-Q x_{1}(t)-r_{1} c(t) x_{1}(t)+\mu_{2} S(t) \\
& -a_{2}\left(Q+r_{2} c(t)\right)-Q x_{2}(t)-r_{2} c(t) x_{2}(t) \\
\geq & \left(\mu_{1}+\mu_{2}\right) S(t)-\sum_{i=1}^{2} a_{i}\left(Q+r_{i} c(t)\right)-\sum_{i=1}^{2}\left(Q+r_{i} c^{*}(0)\right) x_{i}(t) .
\end{aligned}
$$

Integrating both sides of (23) from 0 to $t$ and dividing by $t$ yield

$$
\begin{aligned}
\frac{V(t)}{t}-\frac{V(0)}{t} \geq & \left(\mu_{1}+\mu_{2}\right)\langle S(t)\rangle-\sum_{i=1}^{2} a_{i}\left(Q+r_{i}|c(t)\rangle\right)-\sum_{i=1}^{2}\left(Q+r_{i} c^{*}(0)\right)\left\langle x_{i}(t)\right\rangle \\
\geq & \left(\mu_{1}+\mu_{2}\right) S_{0}-\sum_{i=1}^{2} a_{i}\left(Q+r_{i}|c(t)\rangle\right) \\
& -\sum_{i=1}^{2}\left[\left(Q+r_{i} c^{*}(0)\right)\left(\frac{\mu_{1}+\mu_{2}}{\delta_{i} Q}+1\right)\right]\left\langle x_{i}(t)\right\rangle-\frac{\left(\mu_{1}+\mu_{2}\right) \epsilon(t)}{Q}
\end{aligned}
$$




$$
\begin{aligned}
= & \sum_{i=1}^{2} a_{i}\left(Q+r_{i}\langle c(t)\rangle\right)\left[\frac{\mu_{i} S_{0}}{a_{i}\left(Q+r_{i}\langle c(t)\rangle\right)}-1\right] \\
& -\sum_{i=1}^{2}\left[\left(Q+r_{i} c^{*}(0)\right)\left(\frac{\mu_{1}+\mu_{2}}{\delta_{i} Q}+1\right)\right]\left\langle x_{i}(t)\right\rangle-\frac{\left(\mu_{1}+\mu_{2}\right) \epsilon(t)}{Q} .
\end{aligned}
$$

Notice that $0<S \leq S_{0}$ and $0<x_{i}(t) \leq \delta_{i} S_{0}$. Then $\lim _{t \rightarrow+\infty} \frac{V(t)}{t}=0$ and $\lim _{t \rightarrow+\infty} \epsilon(t)=0$.

Taking the limit inferior of both sides of (24) leads to

$$
\liminf _{t \rightarrow+\infty}\left\langle x_{1}(t)+x_{2}(t)\right\rangle \geq \frac{1}{\Delta_{\max }} \sum_{i=1}^{2} a_{i}\left(Q_{i}+r_{i} \bar{c}\right)\left(\mathcal{R}_{i}-1\right)>0
$$

where

$$
\Delta_{\max }=\max \left\{\left(Q+r_{1} c^{*}(0)\right)\left(\frac{\mu_{1}+\mu_{2}}{\delta_{1} Q}+1\right),\left(Q+r_{2} c^{*}(0)\right)\left(\frac{\mu_{1}+\mu_{2}}{\delta_{2} Q}+1\right)\right\} .
$$

This completes the proof.

\section{Acknowledgements}

The authors would like to thank the anonymous reviewers and the editor for their valuable comments and suggestions that helped to improve the manuscript. This work is supported by the National Natural Science Foundation of China $(11371230,11501331,11561004)$ and Joint Innovative Center for Safe And Effective Mining Technology and Equipment of Coal Resources, Shandong Province, the SDUST Research Fund (2014TDJH102).

\section{Competing interests}

The authors declare that they have no competing interests.

\section{Authors' contributions}

All authors contributed equally to the writing of this paper. All authors read and approved the final manuscript.

\section{Author details}

${ }^{1}$ College of Mathematics and Systems Science, Shandong University of Science and Technology, Qianwangang Road, 279, Qingdao, 266590, P.R. China. ${ }^{2}$ Huangdao Sixteenth Middle School, Zhujing Road, 7, Qingdao, 266000, P.R. China. ${ }^{3}$ State Key Laboratory of Mining Disaster Prevention and Control Co-founded by Shandong Province and the Ministry of Science and Technology, Shandong University of Science and Technology, Qianwangang Road, 279, Qingdao, 266590, P.R. China.

\section{Publisher's Note}

Springer Nature remains neutral with regard to jurisdictional claims in published maps and institutional affiliations.

Received: 6 July 2017 Accepted: 13 September 2017 Published online: 22 September 2017

\section{References}

1. Hsu, SB: A competition model for a seasonally fluctuating nutrient. J. Math. Biol. 9(2), 115-132 (1980)

2. Smith, HL: Competitive coexistence in an oscillating chemostat. SIAM J. Appl. Math. 40(3), 498-522 (1981)

3. Wolkowicz, GSK, Xia, H: Global asymptotic behavior of a chemostat model with discrete delays. SIAM J. Appl. Math. 57(4), 1019-1043 (1997)

4. Meng, X, Gao, Q, Li, Z: The effects of delayed growth response on the dynamic behaviors of the Monod type chemostat model with impulsive input nutrient concentration. Nonlinear Anal., Real World Appl. 11(5), 4476-4486 (2010)

5. Zhang, T, Zhang, T, Meng, X: Stability analysis of a chemostat model with maintenance energy. Appl. Math. Lett. 68, 1-7 (2017)

6. Sun, $M$, Dong, $Q, W u, J$ : Asymptotic behavior of a Lotka-Volterra food chain stochastic model in the chemostat. Stoch. Anal. Appl. 35(4), 645-661 (2017)

7. Zhao, Z, Chen, L: Dynamic analysis of lactic acid fermentation in membrane bioreactor. J. Theor. Biol. 257(2), 270-278 (2009)

8. Hsu, SB, Hubbell, S, Waltman, P: A mathematical theory for single-nutrient competition in continuous cultures of micro-organisms. SIAM J. Appl. Math. 32(2), 366-383 (1977)

9. Zhou, X, Song, X, Shi, X: Analysis of competitive chemostat models with the Beddington-Deangelis functional response and impulsive effect. Appl. Math. Model. 31(10), 2299-2312 (2007)

10. Wang, W, Ma, W, Yan, H: Global dynamics of modeling flocculation of microorganism. Appl. Sci. 6(8), 1-26 (2016) 
11. Guo, S, Ma, W, Zhao, X-Q: Global dynamics of a time-delayed microorganism flocculation model with saturated functional responses. J. Dyn. Differ. Equ. (2017). doi:10.1007/s10884-017-9605-3

12. Guo, S, Ma, W: Global dynamics of a microorganism flocculation model with time delay. Commun. Pure Appl. Anal. 16(5), 1883-1891 (2017)

13. Ma, Z, Cui, G, Wang, W: Persistence and extinction of a population in a polluted environment. Math. Biosci. 101(1), 75-97 (1990)

14. Thomas, DM, Snell, TW, Jaffar, SM: A control problem in a polluted environment. Math. Biosci. 133(2), 139-163 (1996)

15. Dubey, B, Hussain, J: Modelling the interaction of two biological species in a polluted environment. J. Math. Anal. Appl. 246(1), 58-79 (2000)

16. Hallam, TG, Clark, CE, Lassiter, RR: Effects of toxicants on populations: a qualitative approach I. Equilibrium environmental exposure. Ecol. Model. 18(3), 291-304 (1983)

17. Hallam, TG, de Luna, JT: Effects of toxicants on populations: a qualitative. J. Theor. Biol. 109(3), 411-429 (1984)

18. Jiao, J, Ye, K, Chen, L: Dynamical analysis of a five-dimensioned chemostat model with impulsive diffusion and pulse input environmental toxicant. Chaos Solitons Fractals 44(1), 17-27 (2011)

19. Zhang, T, Ma, W, Meng, X: Global dynamics of a delayed chemostat model with harvest by impulsive flocculant input. Adv. Differ. Equ. 2017(1), 115 (2017)

20. Yang, $X, J i n, Z$, Xue, Y: Weak average persistence and extinction of a predator-prey system in a polluted environment with impulsive toxicant input. Chaos Solitons Fractals 31(3), 726-735 (2007)

21. Meng, $X$, Wang, L, Zhang, T: Global dynamics analysis of a nonlinear impulsive stochastic chemostat system in a polluted environment. J. Appl. Anal. Comput. 6(3), 865-875 (2016)

22. Zhao, Z, Chen, L, Song, X: Extinction and permanence of chemostat model with pulsed input in a polluted environment. Commun. Nonlinear Sci. Numer. Simul. 14(4), 1737-1745 (2009)

23. Liu, B, Zhang, L: Dynamics of a two-species Lotka-Volterra competition system in a polluted environment with pulse toxicant input. Appl. Math. Comput. 214(1), 155-162 (2009)

24. Gard, TC: Persistence in stochastic food web models. Bull. Math. Biol. 46(3), 357-370 (1984)

25. Pan, L, Cao, J: Stochastic quasi-synchronization for delayed dynamical networks via intermittent control. Commun. Nonlinear Sci. Numer. Simul. 17(3), 1332-1343 (2012)

26. Zhu, Q, Cao, J: Pth moment exponential synchronization for stochastic delayed Cohen-Grossberg neural networks with Markovian switching. Nonlinear Dyn. 67(1), 829-845 (2012)

27. Liu, M, Bai, C: Analysis of a stochastic tri-trophic food-chain model with harvesting. J. Math. Biol. 73(3), 597-625 (2016)

28. Meng, $X, Z$ Zhao, S, Feng, T, Zhang, T: Dynamics of a novel nonlinear stochastic SIS epidemic model with double epidemic hypothesis. J. Math. Anal. Appl. 433(1), 227-242 (2016)

29. Zhang, Q, Jiang, D, Liu, Z, O'Regan, D: Asymptotic behavior of a three species eco-epidemiological model perturbed by white noise. J. Math. Anal. Appl. 433(1), 121-148 (2016)

30. Zu, L, Jiang, D, O'Regan, D: Conditions for persistence and ergodicity of a stochastic Lotka-Volterra predator-prey model with regime switching. Commun. Nonlinear Sci. Numer. Simul. 29(1), 1-11 (2015)

31. Feng, T, Meng, X, Liu, L, Gao, S: Application of inequalities technique to dynamics analysis of a stochastic eco-epidemiology model. J. Inequal. Appl. 2016(1), 327 (2016)

32. Liu, M, Fan, M: Permanence of stochastic Lotka-Volterra systems. J. Nonlinear Sci. 27(2), 425-452 (2017)

33. Liu, L, Meng, X: Optimal harvesting control and dynamics of two-species stochastic model with delays. Adv. Differ. Equ. 2017(1), 18 (2017)

34. Miao, A, Wang, X, Zhang, T, Wang, W, Sampath Aruna Pradeep, B: Dynamical analysis of a stochastic sis epidemic model with nonlinear incidence rate and double epidemic hypothesis. Adv. Differ. Equ. 2017(1), 226 (2017)

35. $\mathrm{Ma}, \mathrm{H}, \mathrm{Hou}, \mathrm{T}$ : A separation theorem for stochastic singular linear quadratic control problem with partial information. Acta Math. Appl. Sin. Engl. Ser. 29(2), 303-314 (2013)

36. Liu, X, Li, Y, Zhang, W: Stochastic linear quadratic optimal control with constraint for discrete-time systems. Appl. Math. Comput. 228, 264-270 (2014)

37. Miao, A, Zhang, J, Zhang, T, Pradeep, BGS: Threshold dynamics of a stochastic SIR model with vertical transmission and vaccination. Comput. Math. Methods Med. 2017, 4820183 (2017)

38. Ma, H, Jia, Y: Stability analysis for stochastic differential equations with infinite Markovian switchings. J. Math. Anal. Appl. 435(1), 593-605 (2016)

39. Liu, Q, Jiang, D, Shi, N, Hayat, T, Alsaedi, A: Stochastic mutualism model with Lévy jumps. Commun. Nonlinear Sci. Numer. Simul. 43, 78-90 (2017)

40. Zhao, Y, Zhang, W: Observer-based controller design for singular stochastic Markov jump systems with state dependent noise. J. Syst. Sci. Complex. 29(4), 946-958 (2016)

41. Gu, Y, Ren, Y, Sakthivel, R: Square-mean pseudo almost automorphic mild solutions for stochastic evolution equations driven by g-Brownian motion. Stoch. Anal. Appl. 34(3), 528-545 (2016)

42. Liu, Y, Liu, Q, Liu, Z: Dynamical behaviors of a stochastic delay logistic system with impulsive toxicant input in a polluted environment. J. Theor. Biol. 329, 1-5 (2013)

43. Liu, M, Wang, K: Persistence and extinction of a single-species population system in a polluted environment with random perturbations and impulsive toxicant input. Chaos Solitons Fractals 45(12), 1541-1550 (2012)

44. Zhu, Q, Cao, J: Robust exponential stability of Markovian jump impulsive stochastic Cohen-Grossberg neural networks with mixed time delays. IEEE Trans. Neural Netw. 21(8), 1314-1325 (2010)

45. Sakthivel, R, Luo, J: Asymptotic stability of nonlinear impulsive stochastic differential equations. Stat. Probab. Lett. 79(9), 1219-1223 (2009)

46. Yang, X, Cao, J, Lu, J: Stochastic synchronization of complex networks with nonidentical nodes via hybrid adaptive and impulsive control. IEEE Trans. Circuits Syst. I, Regul. Pap. 59(2), 371-384 (2012)

47. Zhang, $S$, Meng, $X$, Feng, $T$, Zhang, T: Dynamics analysis and numerical simulations of a stochastic non-autonomous predator-prey system with impulsive effects. Nonlinear Anal. Hybrid Syst. 26, 19-37 (2017)

48. Ren, Y, Jia, X, Sakthivel, R: The p-th moment stability of solutions to impulsive stochastic differential equations driven by g-Brownian motion. Appl. Anal. 96(6), 988-1003 (2017)

49. Diop, MA, Rathinasamy, S, Ndiaye, AA: Neutral stochastic integrodifferential equations driven by a fractional Brownian motion with impulsive effects and time-varying delays. Mediterr. J. Math. 13(5), 2425-2442 (2016) 
50. Liu, M, Wang, K: Persistence and extinction of a stochastic single-specie model under regime switching in a polluted environment. J. Theor. Biol. 264(3), 934-944 (2010)

51. Chen, L, Chen, J: Nonlinear Biological Dynamical System. Science Press, Beijing (1993)

52. Mao, X: Stochastic Differential Equations and Applications. Horwood, Chichester (2007)

53. Liu, M, Wang, K: Persistence and extinction of a stochastic single-species population model in a polluted environment with impulsive toxicant input. Electron. J. Differ. Equ. 2013, 230 (2013)

54. Sun, S, Sun, Y, Zhang, G, Liu, X: Dynamical behavior of a stochastic two-species Monod competition chemostat model. Appl. Math. Comput. 298, 153-170 (2017)

Submit your manuscript to a SpringerOpen ${ }^{\odot}$ journal and benefit from:

- Convenient online submission

- Rigorous peer review

- Open access: articles freely available online

- High visibility within the field

- Retaining the copyright to your article

Submit your next manuscript at $\gg$ springeropen.com 